\title{
CARCINOMA HEPATOCELULAR: impacto do tempo em lista e das formas de tratamento pré-operatório na sobrevida do transplante de fígado cadavérico na era pré-MELD em um centro no Brasil
}

\author{
Alexandre Coutinho Teixeira de FREITAS, Mônica Beatriz PAROLIN, \\ Lucinei STADNIK, Júlio Cezar Uili COELHO
}

RESUMO - Racional - Atualmente, o transplante hepático é a principal opção terapêutica para doentes com cirrose hepática associada a carcinoma hepatocelular. Objetivos - Analisar a sobrevida em 3 meses e 1 ano de pacientes com e sem carcinoma hepatocelular submetidos a transplante hepático cadavérico. Métodos - Foram revisados os prontuários dos pacientes submetidos a transplante hepático cadavérico no Hospital de Clínicas da Universidade Federal do Paraná no período entre 5 de janeiro de 2001 e 17 de fevereiro de 2006. Os pacientes foram divididos em 2 grupos - acometidos e não-acometidos de carcinoma hepatocelular - e analisados em relação à sobrevida em 3 meses e em 1 ano. Também foram comparados em relação ao sexo e à idade do doador e do receptor, a causa da cirrose, a classificação de Child-Pugh e o escore do MELD no momento do transplante, o tempo de isquemia morna e isquemia fria, o número de unidades de concentrado de hemácias transfundidas durante o transplante, o tempo de permanência na UTI e o tempo de internação. Resultado - Foram analisados 146 casos de transplante hepático: 75 foram excluídos devido a dados incompletos no prontuário e 71 foram incluídos no estudo. A sobrevida geral em 3 meses e 1 ano foi de 77,4\% e 74,6\%, respectivamente. Os acometidos por carcinoma hepatocelular $(n=12)$ apresentaram sobrevida em 3 meses e 1 ano de 100\%, significantemente maior que os não-acometidos ( $n=59 ; 72,8 \%$ e 69,49\%, respectivamente). O índice médio do MELD, da classificação de Child-Pugh e o número médio de concentrado de hemácias transfundidas foram significantemente maiores nos pacientes não-acometidos. Também foi observada maior percentagem de pacientes classificados como Child-Pugh $\mathrm{B}$ e $\mathrm{C}$ e de pacientes com diagnóstico de cirrose por outras causas nos pacientes não acometidos pela neoplasia. Nos doentes com carcinoma hepatocelular foi observada maior percentagem de indivíduos classificados como Child-Pugh A e com diagnóstico de cirrose por hepatite C. Todos os outros fatores analisados foram iguais entre os 2 grupos. Conclusão - Os doentes com carcinoma hepatocelular, submetidos a transplante hepático cadavérico, apresentam maior sobrevida em 3 meses e 1 ano do que os não acometidos por esta neoplasia. Essa diferença é possivelmente relacionada à realização do transplante nos pacientes com carcinoma hepatocelular em estádio menos avançado da cirrose.

DESCRITORES - Carcinoma hepatocelular. Cirrose hepática. Transplante de fígado. Análise de sobrevida.

\section{INTRODUÇÃO}

O carcinoma hepatocelular (CHC) é a sétima causa de óbito relacionada à neoplasia ${ }^{(15)}$. Sua incidência aumentou em $71 \%$ nos Estados Unidos, no período de 20 anos, compreendido entre 1976 e 1995, passando de 1,4/100.000 para 2,4/100.000 habitantes ${ }^{(4,6)}$. Em aproximadamente $90 \%$ dos casos existe infecção pelos vírus da hepatite B ou C. O aumento da incidência do CHC está relacionado ao aumento da incidência de hepatite $\mathrm{C}$ observado no mesmo período ${ }^{(8)}$. Pacientes cirróticos com $\mathrm{CHC}$ são freqüentemente tratados através do transplante hepático (Tx), com potencial de cura para ambas as doenças ${ }^{(15}$, 16. Existe grande preocupação relacionada à sobrevida desses pacientes. A presença de neoplasia associada à imunossupressão no período pós-transplante apresenta risco de disseminação local e à distância, com resultados desastrosos. Isso foi confirmado através dos estudos iniciais sobre o Tx, em que a recurrência da neoplasia ocorreu em $32 \%$ a $54 \%$ dos casos e a sobrevida em 5 anos foi menor do que $40 \%{ }^{(18)}$. Esses estudos serviram de embasamento para determinação de limites à indicação de Tx em doentes com CHC. Atualmente, resultados excelentes em termos de sobrevida são obtidos para pacientes com nódulos solitários até $5 \mathrm{~cm}$ ou até três nódulos menores que $3 \mathrm{~cm}^{(1,13,14)}$.

O objetivo desse estudo foi analisar a sobrevida de pacientes cirróticos com $\mathrm{CHC}$ submetidos a Tx cadavérico em relação a pacientes submetidos a transplante por outras indicações.

Serviço de Transplante Hepático do Hospital de Clínicas da Universidade Federal do Paraná, Curitiba, PR.

Correspondência: Dr. Alexandre C. Teixeira de Freitas - Rua Brasílio Itiberê, 4029 - apt. 52 - 80240-060 - Curitiba, PR. E-mail: alexandrefreitas@ufpr.br 


\section{MÉTODOS}

Foram revisados os prontuários dos pacientes submetidos a Tx cadavérico no Hospital de Clínicas da Universidade Federal do Paraná, Curitiba, PR, no período entre 5 de janeiro de 2001 e 17 de fevereiro de 2006. As seguintes informações do receptor foram coletadas: sexo, idade, causa da cirrose, presença ou não de CHC, sobrevida do paciente em 3 meses e em 1 ano, classificação de Child-Pugh no momento do transplante, escore do MELD no momento do transplante, tempo de isquemia morna, número de unidades de concentrado de hemácias transfundidas durante o transplante, tempo de permanência na UTI e tempo de internação. Em relação ao doador, foram coletados dados referentes ao sexo, idade e ao tempo de isquemia fria. Foram excluídos pacientes com dados incompletos no prontuário.

Os pacientes em lista de espera para transplante cadavérico foram submetidos a rastreamento para CHC a cada 6 meses, através de ecografia abdominal e dosagem de alfa-fetoproteína. O diagnóstico de $\mathrm{CHC}$ foi realizado através dos critérios de Barcelona ${ }^{(2,3)}$ : a) para nódulos maiores que $2 \mathrm{~cm}$, através da associação de dois exames de imagem - tomografia, ressonância magnética ou arteriografia - demonstrando hipervascularização arterial no nódulo ou um exame de imagem demonstrando hipervascularização arterial associado à alfa-fetoproteína maior que $400 \mu \mathrm{g} / \mathrm{L}$; b) para nódulos entre $1 \mathrm{~cm}$ e $2 \mathrm{~cm}$, através de biopsia hepática guiada - nos casos com resultado negativo para malignidade, os pacientes foram submetidos a seguimento com exames de imagem; c) para nódulos menores que $1 \mathrm{~cm}$, seguimento com exames de imagem. Pacientes com diagnóstico de $\mathrm{CHC}$ foram submetidos a tratamento com quimioembolização ou alcoolização, dependendo da localização, número de lesões e disponibilidade do método. OTx foi realizado somente nos pacientes que apresentaram nódulo único com diâmetro menor ou igual a $5 \mathrm{~cm}$ ou até três nódulos menores ou iguais a $3 \mathrm{~cm}$ cada. Não foram transplantados pacientes com critérios expandidos, que permaneceram em terapia com alcoolização ou quimioembolização, ou foram encaminhados para quimioterapia sistêmica. Foram analisados o tempo entre o diagnóstico de $\mathrm{CHC}$ e a realização do transplante, o número e a localização dos nódulos, o tipo de tratamento local realizado, o número de sessões, o maior diâmetro dos nódulos nos exames de imagem no momento do diagnóstico, nos últimos exames de imagem antes do transplante e no exame anatomopatológico do explante.

Foi realizada análise univariada da sobrevida - em 3 meses e 1 ano - de acordo com a divisão dos pacientes em 2 grupos: a) grupo 1 - não-acometidos de $\mathrm{CHC}$; e b) grupo 2 - acometidos de CHC. Esses dois grupos também foram analisados em relação à idade média e sexo, causa da cirrose, número médio de unidades de concentrado de hemácias transfundidas, tempo médio de permanência na UTI, tempo médio de internação, idade média e sexo do doador e o tempo médio de isquemia fria. Para análise estatística, em relação à causa da cirrose, os pacientes foram divididos em três grupos: grupo 1 - doença colestática; grupo 2 - hepatite $\mathrm{C}$; grupo 3 - outras causas. Nas doenças colestáticas foram incluídos os casos de cirrose biliar primária, colangite esclerosante primária, cirrose biliar secundária e doenças hepáticas císticas. Nas outras causas foram incluídos casos de cirrose alcoólica, hepatite auto-imune, cirrose criptogênica, hepatite $\mathrm{B}$, polineuropatia amiloidótica e associação de cirrose alcoólica e hepatite B.

Para análise do maior diâmetro médio dos nódulos foi utilizado o teste $t$. Para a análise univariada relacionada à sobrevida, foi utilizado o método de Kaplan-Meier com análise estatística pelo teste de Log-Rank. Para a análise comparativa dos dados entre acometidos e não-acometidos de $\mathrm{CHC}$ foi utilizado o teste $t$ para médias e o teste do qui-quadrado para proporções. Foi considerado o nível de significância de $5 \%(P \leq 0,05)$.

\section{RESULTADOS}

Foram analisados 146 casos de Tx, dos quais 75 foram excluídos devido a dados incompletos no prontuário e 71 foram incluídos no estudo: a) grupo 1 - não-acometidos de $\mathrm{CHC}(\mathrm{n}=59)$; b) grupo 2 - pacientes com $\mathrm{CHC}(\mathrm{n}=12)$. Não houve diferença em relação à idade média dos receptores e doadores nos dois grupos: 48,6 \pm 13 anos e 30,3 \pm 13 anos nos doentes com CHC e de $54,1 \pm 7$ anos e $34,6 \pm 12$ anos nos não-acometidos. Os dois grupos foram iguais em relação ao sexo dos doadores e receptores, tempo médio de permanência na UTI, tempo médio de internação, tempo médio de isquemia fria e de isquemia morna. A Tabela 1

TABELA 1. Dados referentes aos receptores

\begin{tabular}{|c|c|c|c|c|}
\hline & Total & Com HCC & Sem HCC & $P$ \\
\hline $\mathrm{n}$ & 71 & 12 & 59 & - \\
\hline \multicolumn{5}{|l|}{ Causa da cirrose } \\
\hline Doenças colestáticas (CBP) & $4(5,6 \%)$ & 0 & $4(6,8 \%)$ & 0,05 \\
\hline Hepatite C & $31(43,7 \%)$ & $9(75 \%)$ & $22(37,3 \%)$ & 0,05 \\
\hline Outras causas & $36(50,7 \%)$ & $3(25 \%)$ & $33(55,9 \%)$ & 0,05 \\
\hline Escore médio MELD & $15,1 \pm 4$ & $11,4 \pm 2$ & $15,8 \pm 4$ & $<0,01$ \\
\hline \multicolumn{5}{|l|}{ Classificação de Child-Pugh } \\
\hline Escore médio & $8,4 \pm 1$ & $6,7 \pm 1$ & $8,7 \pm 1$ & $<0,01$ \\
\hline Child A & $6(8,5 \%)$ & $4(33,3 \%)$ & $2(3,4 \%)$ & $<0,01$ \\
\hline Child B & $49(69,1 \%)$ & $8(66,6 \%)$ & $41(69,4 \%)$ & \\
\hline Child C & $16(22,4 \%)$ & 0 & $16(27,2 \%)$ & \\
\hline Média de concentrado de hemácias transfundidas (unidades) & $4,6 \pm 3$ & $2,9 \pm 2$ & $5 \pm 3$ & 0,03 \\
\hline
\end{tabular}


contém dados referentes aos receptores. Os dois grupos foram diferentes em relação à causa da cirrose. No grupo 1 foi observada maior percentagem de pacientes com diagnóstico de cirrose de outras causas; no grupo 2 foi observada maior percentagem de pacientes com diagnóstico de hepatite $\mathrm{C}$. O índice médio do MELD foi significantemente maior nos pacientes não-acometidos por CHC (grupo 1), o mesmo ocorrendo com o índice da classificação de Child-Pugh. Nos não-acometidos por CHC observou-se maior percentagem de pacientes classificados como Child-Pugh B e C e nos acometidos por $\mathrm{CHC}$ maior percentagem de classificados como Child-Pugh A. A média de concentrado de hemácias transfundidas foi maior no grupo 1 em relação ao grupo 2 .

O tempo médio decorrido entre o diagnóstico de $\mathrm{CHC}$ e a realização do Tx foi de 19,3 \pm 16 meses (variação de 1 até 48 meses). No total foram detectados 21 nódulos: em cinco pacientes foi detectado 1 nódulo, em cinco foram detectados 2 e em dois, 3. Em 11 casos estes estavam localizados somente no lobo hepático direito, em 1 caso havia apenas um no lobo direito e outro no lobo esquerdo. O diâmetro médio dos nódulos foi de $3,3 \pm 1 \mathrm{~cm}$ no momento do diagnóstico. Oito pacientes foram submetidos a média de 2,5 sessões de quimioembolização (variação de 1 até 6 sessões). Dois pacientes foram submetidos a quimioembolização ( 2 e 3 sessões) associada à alcoolização ( 5 sessões cada um). Os últimos exames de imagem realizados antes do transplante, mostraram remissão total dos nódulos em três casos com nódulo único. Houve diminuição do diâmetro médio dos nódulos para $2,4 \pm 1 \mathrm{~cm}(P=0,02)$ após a terapia local. Dois pacientes não foram submetidos a terapia local: o primeiro porque o transplante foi realizado 1 mês após o diagnóstico de $\mathrm{CHC}$, o segundo porque a biopsia hepática realizada no momento da deteç̧ão de dois nódulos foi negativa. Esse paciente foi submetido a acompanhamento com exames de imagem seriados, havendo crescimento de $2 \mathrm{~cm}$ no diâmetro de um dos nódulos nos 33 meses de seguimento até o transplante. $\mathrm{O}$ exame anatomopatológico do explante mostrou carcinoma $\mathrm{CHC}$ em um nódulo e colangiocarcinoma no outro. O exame anatomopatológico dos outros pacientes confirmou o diagnóstico de $\mathrm{CHC}$ em oito casos. Os nódulos apresentaram diâmetro médio de 2,8 $\pm 1 \mathrm{~cm}$, sem diferença estatística em relação ao diâmetro no momento do diagnóstico $(P=0,13)$ e em relação ao diâmetro após a terapia local $(P=0,16)$. Em três casos observou-se remissão completa. Invasão angiolinfática foi observada em um caso. Invasão capsular não foi observada nos casos analisados. Dos oito casos de CHC, dois foram descritos como grau III da classificação de Edmondson-Steiner e seis como grau II.

A sobrevida geral em 3 meses e 1 ano (Figuras 1 e 2) foi de $77,4 \%$ e 74,6\%, respectivamente. Os pacientes com CHC apresentaram sobrevida em 3 meses significantemente maior que os não-acometidos $(100 \%$ e $72,8 \%$, respectivamente, $P=0,04)$, conforme a Figura 3. O mesmo aconteceu em relação à sobrevida em 1 ano $(100 \%$ e 69,4\% respectivamente, $P=0,02)$, conforme a Figura 4 .

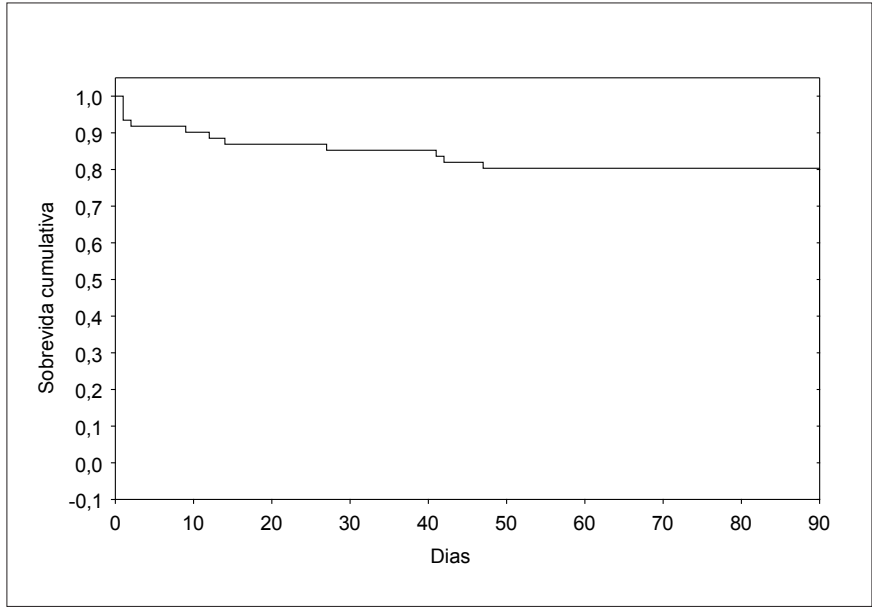

FIGURA 1. Sobrevida cumulativa em 3 meses

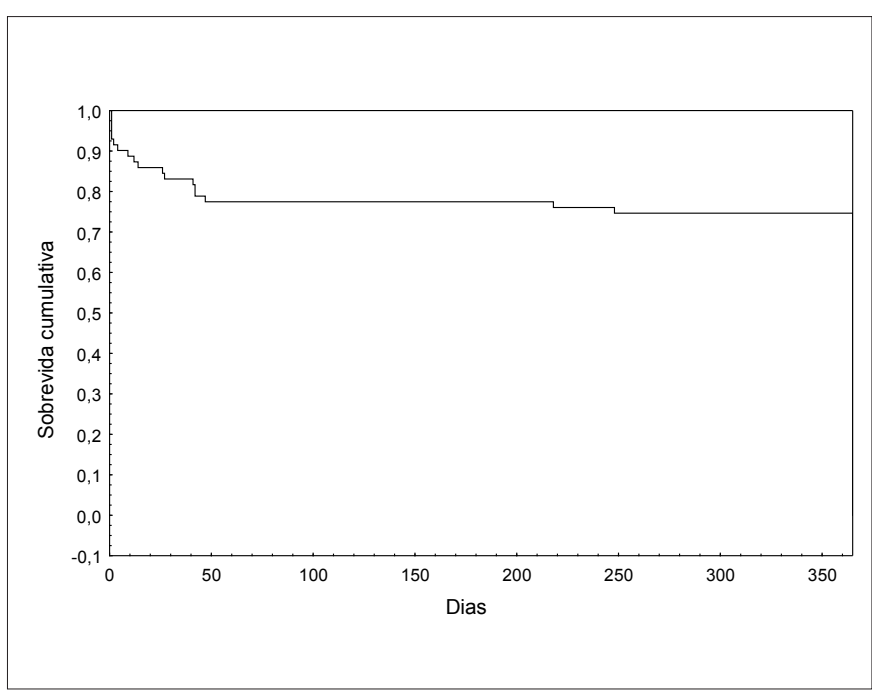

FIGURA 2. Sobrevida cumulativa em 1 ano

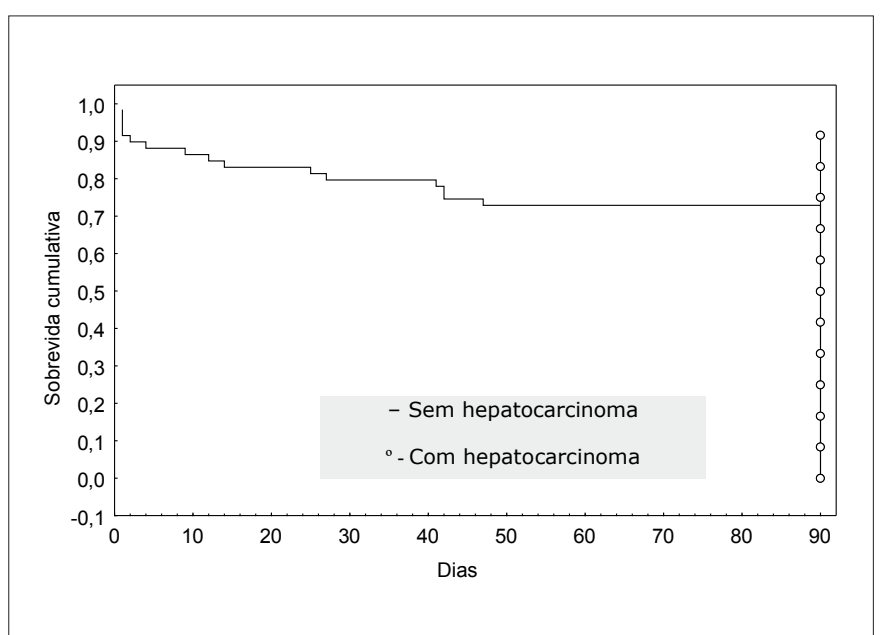

FIGURA 3. Sobrevida cumulativa em 3 meses de acometidos e nãoacometidos de carcinoma hepatocelular 


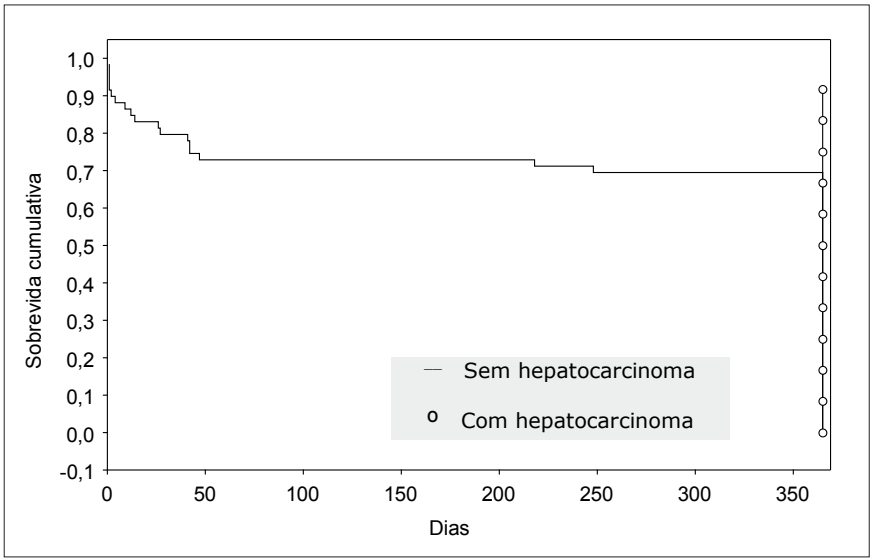

FIGURA 4. Sobrevida cumulativa em 1 ano de acometidos e não-acometidos de carcinoma hepatocelular

\section{DISCUSSÃO}

O Tx é atualmente a melhor alternativa para tratamento da cirrose hepática associada ao $\mathrm{CHC}$, devido a possibilidade de resolução das duas doenças ${ }^{(11)}$. A remoção do fígado doente evita a ocorrência de tumores sincrônicos e metacrônicos. Inicialmente, os critérios para indicação de transplante em acometidos por $\mathrm{CHC}$ eram mais amplos, o que gerou resultados ruins em termos de sobrevida. Atualmente, o critério mais aceito para indicação é restrito a pacientes com doença precoce: nódulo único até $5 \mathrm{~cm}$ de diâmetro ou até três nódulos com até $3 \mathrm{~cm}$ cada um ${ }^{(1,14)}$.

Nessa situação, diversos estudos na literatura têm demonstrado resultados que variam de $70 \%$ a $90 \%$ para a sobrevida em 1 ano e de $49 \%$ a $75 \%$ para a sobrevida em 5 anos s $^{(1,7,8,10,13,21)}$. Segundo dados da United Network for Organ Sharing - UNOS, a sobrevida em 1 ano de doentes com CHC é igual a de não-acometidos ${ }^{(20)}$. As estatísticas envolvendo os transplantes realizados entre $2002 \mathrm{e}$ 2004, mostram sobrevida em 1 ano de 86,3\% em 1.066 pacientes cirróticos com doença maligna e de $85,8 \%$ em 6.543 pacientes com cirrose hepática não-colestática. No entanto, há diferença da sobrevida em 3 anos dos transplantes realizados entre 1999 e 2002 e da sobrevida em 5 anos dos transplantes realizados entre 1997 e 2000 . Em 491 doentes com cirrose e doença maligna a sobrevida em 3 anos foi de $69,8 \%$, significantemente menor que a sobrevida de $76,3 \%$ observada em 6.983 pacientes com cirrose de origem não-colestática. A sobrevida em 5 anos foi de $57,6 \%$ em 265 pacientes com cirrose e doença maligna e de $69,2 \%$ em 5.218 com cirrose não-colestática.

Neste estudo observou-se sobrevida geral de $74,6 \%$ em 1 ano. A análise dos doentes com CHC mostrou sobrevida de $100 \%$, significantemente maior em relação aos não-acometidos. A análise dos dois grupos demonstrou diferença no estádio evolutivo da cirrose hepática através do escore do MELD e da classificação de Child-Pugh. Nos acometidos de CHC eles foram significantemente menores em relação aos outros pacientes. $\mathrm{O}$ diagnóstico de $\mathrm{CHC}$, ainda que em fase evolutiva precoce da cirrose sem comprometimento importante da função hepática, foi o motivo da listagem desses pacientes. Os não-acometidos de $\mathrm{CHC}$ foram listados mais tardiamente, somente após a verificação de evolução da cirrose, apresentando número significativo de pacientes na classe $\mathrm{C}$ de Child e com índice médio do MELD mais elevados. $\mathrm{O}$ transplante precoce nos doentes com $\mathrm{CHC}$ proporcionou ainda menor necessidade de transfusão sangüínea no peroperatório, fator esse relacionado intimamente com o grau de hipertensão portal e de alteração de fatores de coagulação.

Pacientes com escore do MELD mais baixos ou com classificação de Child-Pugh A ou B apresentam melhor prognóstico após a realização de $\mathrm{Tx}^{(5,21)}$. Em um estudo, 1.472 pacientes foram estratificados de acordo com o índice do MELD em três grupos: a) MELD de 6 a 15; b) MELD 16 a 25; c) MELD maior que $25^{(5)}$. A sobrevida em 1 ano e em 10 anos foi de $86 \%, 84 \%$, $75 \%$ e $62 \%, 59 \%, 45 \%$, respectivamente, havendo diferença significante entre os grupos. Outro estudo com 8.102 pacientes demonstrou que incremento de 10 pontos no escore do MELD está associado ao aumento de 39\% na mortalidade em 1 ano após o transplante ${ }^{(9)}$. A vantagem conferida pela realização do transplante em fase mais precoce da doença reflete-se na sobrevida em 1 ano. No entanto, após 3 anos, pacientes com CHC apresentam diminuição da sobrevida relacionada à recidiva da neoplasia ou metástases à distância ${ }^{(19)}$. A sobrevida livre de recidiva da neoplasia está relacionada ao estádio pós-operatório realizado na análise patológica do explante ${ }^{(19)}$. Estudo mostrou que $43 \%$ dos pacientes apresentaram-se fora dos critérios de Milão na análise do explante, a despeito da análise pré-operatória com exames de imagem incluir os doentes nesses critérios ${ }^{(19)}$. A sobrevida livre de neoplasia em 3 anos foi de $67 \%$, significantemente menor que a sobrevida dos pacientes que foram classificados dentro dos critérios de Milão no pós-operatório $(87 \%)^{(19)}$.

A grande dificuldade é a obtenção de um órgão em estádio evolutivo precoce da cirrose. No Brasil, até recentemente, na era pré-MELD, os critérios de alocação de órgãos privilegiavam os receptores pela ordem de inscrição na lista de espera. Devido à carência de órgãos no país, o período de espera nessa situação era prolongado, ocorrendo progressão da cirrose e do CHC e realização do transplante em condições menos favoráveis. $\mathrm{O}$ risco de progressão do $\mathrm{CHC}$ durante o período em lista de espera, ultrapassando-se os critérios para indicação do transplante varia, em alguns estudos, de $20 \%$ a $50 \%{ }^{(2,10)}$. Segundo dados da Associação Brasileira de Transplantes de Órgãos -ABTO, no ano de 2005 foram realizados no Brasil 956 Tx $^{(17)}$. Destes, 763 nos Estados de São Paulo, Rio de Janeiro, Paraná, Rio Grande do Sul e Pernambuco. No entanto, a lista de espera em janeiro de 2006 nesses Estados era de 6.501 pacientes. Em uma estimativa simplista, o período de espera seria de 8,5 anos. Atualmente, com a utilização do MELD como critério de distribuição de órgãos, a probabilidade de realização de transplante em fase favorável de evolução da cirrose é maior. Esse sistema prevê a necessidade mais urgente de um órgão nos doentes com $\mathrm{CHC}$, conferindo pontuação extra no índice do MELD.

Nos Estados Unidos, o uso do MELD como critério de distribuição de órgãos elevou o número de transplantes em doentes com CHC de $3,1 \%$ para $22,2 \%{ }^{(9)}$. No entanto, apesar do transplante ocorrer em fase mais precoce da evolução da 
cirrose, a sobrevida em 1 ano de acometidos de $\mathrm{CHC}$ foi igual a dos não-acometidos, tanto na era pré-MELD quanto na era pós-MELD. Infelizmente os autores não testaram a sobrevida dos doentes com CHC na era pré-MELD em relação a era pós-MELD.

Terapia local com alcoolização, quimioembolização, ablação por radiofreqüência, entre outras, pode diminuir a progressão da neoplasia ${ }^{(13)}$. Isso permite período maior de espera em lista. Observou-se nesse estudo, estabilização da doença com a utilização de quimioembolização ou quimioembolização associada à alcoolização. Inclusive, em três casos, houve remissão completa da doença evidenciada ao exame anatomopatológico do explante. Dos 12 pacientes com hepatocarcinoma, 8 apresentaram classificação de Child-Pugh B e 4 Child-Pugh A. Estes, apesar da pontuação baixa nessa classificação, apresentavam sinais de hipertensão porta. Este motivo, associado ao fato desses quatro pacientes terem hepatite $\mathrm{C}$ e de os nódulos não serem periféricos, motivou a realização do Tx ao invés de ressecção após a boa evolução decorrente de terapia local.
OTx intervivos é alternativa muito utilizada no Brasil devido ao longo tempo em lista de espera. Em 2005, dos 956 transplantes hepáticos realizados, 197 (20,6\%) foram com doador vivo ${ }^{(16)}$. O Brasil é atualmente um dos países com maior experiência na realização desse procedimento no mundo. Nos Estados Unidos, em 2005, dos 6.443 transplantes realizados, 6.120 foram cadavéricos e $323(5 \%)$ foram intervivos ${ }^{(20)}$. Doentes com CHC se beneficiam do curto tempo de espera em lista, não havendo necessidade de terapia local em muitos casos. Grande limitação é imposta pelo risco ao doador. Espera-se que, com a introdução do MELD como critério de alocação de órgãos no Brasil, ocorra incremento do número de transplantes cadavéricos realizados para doentes com $\mathrm{CHC}$, à semelhança do que ocorreu nos Estados Unidos, diminuindo a necessidade de transplante intervivos.

Os resultados do presente estudo indicam que pacientes cirróticos, acometidos de CHC, apresentam maior sobrevida em 3 meses e em 1 ano em relação aos não-acometidos. Isso está associado à realização do transplante em estádio de evolução mais precoce da cirrose.

Freitas ACT, Parolin MB, Stadnik L, Coelho JCU. Hepatocellular carcinoma: impact of waiting list and pre-operative treatment strategies on survival of cadaveric liver transplantation in pre-MELD era in one center in Brazil. Arq Gastroenterol. 2007;44(3):189-94.

ABSTRACT - Background - Liver transplantation is the main treatment option for hepatocellular carcinoma in patients with cirrhosis. Aim - Three months and 3 years survival were analysed in patients with cirrhosis and hepatocellular carcinoma and in patients with only cirrhosis. Methods - Charts of patients subjected to cadaveric liver transplantation at the Clinical Hospital of the Federal University of Paraná, Curitiba, PR, Brazil, between January 5th of 2001 and February 17th of 2006 were reviewed. Patients were divided into two groups for 3 months and 1 year survival analysis: cirrhosis and hepatocellular carcinoma and cirrhosis only. The two groups were also compared in relation to donor and recipient sex and age, etiology of cirrhosis, Child-Pugh and MELD scores at the time of the transplantation, warm isquemia time, cold isquemia time, units of red blood cells transfused during the transplantation, intensive care unit stay and total hospital stay. Results - One hundred and forty six liver transplantation patients were analysed: 75 were excluded because of incomplete data and 71 were included. General 3 months and 1 year survivals were 77,4\% and 74,6\% respectively. Patients with hepatocellular carcinoma $(\mathrm{n}=12)$ presented 3 months and 1 year survivals of $100 \%$. These rates were significantly higher than those of patients without hepatocellular carcinoma $(\mathrm{n}=59 ; 72,8 \%$ and 69,4\%). Mean MELD score, mean Child-Pugh score and mean number of red blood cells transfused were significantly higher in patients without hepatocellular carcinoma. In this group it was also observed more Child-Pugh B and C patients and the diagnosis of cirrhosis because other causes. The rate of Child-Pugh $\mathrm{A}$ and hepatitis $\mathrm{C}$ was higher in patients with hepatocellular carcinoma. The two groups were identical in all other parameters analysed. Conclusion - Patients with cirrhosis and hepatocellular carcinoma presented better 3 months and 1 year survival rates than patients with only cirrhosis. This is possibly due to an early stage of cirrhosis at transplantation of patients with hepatocellular carcinoma.

HEADINGS - Carcinoma, hepatocellular. Liver cirrhosis. Liver transplantation. Survival analysis. 


\section{REFERÊNCIAS}

1. Bismuth H, Majno PE, Adam R. Liver transplantation for hepatocellular carcinoma. Semin Liver Dis. 1999;19:311-22.

2. Bruix J, Castells A, Bosch J, Feu F, Fuster J, Garcia-Pagan JC, Visa J, Bru C, Rodes J. Surgical resection of hepatocellular carcinoma in cirrhotic patients: prognostic value of preoperative portal pressure. Gastroenterology. 1996;111:1018-22.

3. Bruix J, Sherman M, Llovet JM, Beaugrand M, Lenciono R, Burroughs AK, Christensen E, Pagliaro L, Colombo M, Rodes J, EASL Panel of Experts of HCC. Clinical management of hepatocellular carcinoma: conclusions of the Barcelona-2000 EASL Conference. J Hepatol. 2001;35:421-30.

4. El-Serag HB, Mason AC. Rising incidence of hepatocellular carcinoma in the United States. N Engl J Med. 1999;340:745-50.

5. Habib S, Berk b, Chang CH, Demetris AJ, Fontes P, Dvorchik I, Eghtesad B, Marcos A, Shakil AO. MELD and prediction of post-liver transplantation survival. Liver Transplant. 2006;12:440-7.

6. Ikeda K, Saitoh S, Koida I, Arase Y, Tsubota A, Chayama K, Kumada H, Kawanishi M. A multivariate analysis of risk factors for hepatocellular carcinogenesis: a prospective observation of 795 patients with viral and alcoholic cirrhosis. Hepatology. 1993;18:47-53.

7. Iwatsuki S, Starzl TE, Sheahan DG, Yokoyama I, Demetris AJ, Todo S, Tzakis AG, Van Thiel DH, Carr B, Selby R, et al. Hepatic resection versus transplantation for hepatocellular carcinoma. Ann Surg. 1991;214:221-8.

8. Jonas S, Bechstein WO, Steinmuller T, Hermann M, Radke C, Berg T, Settmacher U, Neuhaus P. Vascular invasion and histolopathologic grading determine outcome after liver transplantation for hepatocellular carcinoma in cirrhosis. Hepatology. 2001;33:1080-6.

9. Kanwal F, Dulai GS, Spiegel BM, Yee HF, Gralnek IM. A comparison of liver transplantation outcomes in the pre- vs Post-MELD eras. Aliment Pharmacol Ther. 2005;21:169-77.

10. Llovet JM, Fuster J, Bruix J. Intention-to-treat analysis of surgical treatment for early hepatocellular carcinoma: resection versus transplantation. Hepatology. 1999;39:1434-40
11. Llovet JM, Burroughs A, Bruix J. Hepatocellular Carcinoma. Lancet 2003;362:1907-17.

12. Majno PE, Adam R, Bismuth H, Castaing D, Ariche A, Krissat I, Perrin H, Azoulay D. Influence of preoperative transarterial lipiodol chemoembolization on resection and transplantation for hepatocellular carcinoma in patients with cirrhosis. Ann Surg. 1997;226:688-701.

13. Mazzaferro V, Regalia E, Doci R, Andreola S, Pulvirenti A, Bozzetti F, Montalto F, Ammatuna M, Morabito A, Gennari L. Liver transplantation for the treatment of small hepatocellular carcinoma in patients with cirrhosis. N Engl J Med 1996;334:693-9.

14. Mor E, Kaspa RT, Sheiner P, Schwartz M. Treatment of hepatocellular carcinoma associated with cirrhosis in the era of liver transplantation. Ann Intern Med 1998; 129:643-53.

15. Okuda K, Ohtsuki T, Obata H, Tomimatsu M, Okazaki N, Hasegawa H, Nakajima Y, Onishi K. Natural history of hepatocellular carcinoma and prognosis in relation to treatment. Study of 850 patients. Cancer 1985;56:918-28.

16. Okuda K. Hepatocellular carcinoma: recent progress. Hepatology 1992;15:948-63.

17. Registro Brasileiro de Transplantes. 2005;11(2) Disponível em http://www.abto org.br

18. Ringe B, Pichlmayr R, Wittekind C, Tursch G. Surgical treatment of hepatocellular carcinoma: experience with liver resection and transplantation in 198 patients. World J Surg. 1991:15:270-85.

19. Shah SA, Tan JC, McGilvray ID, Cattral MS, Cleary SP, Levy GA, Greig PD, Gran DR. Accuracy of staging as a predictor for recurrence after liver transplantation for hepatocellular carcinoma. Transplantation 2006;81:1633-9.

20. United Network for Organ Sharing. 2005 Annual report of the U.S. scientific registry for transplant recipients and the organ procurement and transplantation network. Available at: www.optn.org.

21. Yoo HY, Thuluvath PJ. Short-term post liver transplant survival after the introduction of MELD scores for organ allocation in the United States. Liver Int. 2005;25:536-41.

Recebido em 22/9/2006 Aprovado em 8/6/2007. 